\title{
Explorando los conceptos de polígonos y poliedros desde el modelo de Van Hiele
}

\section{Exploring the concepts of polygons and polyhedra from Van Hiele's model}

\author{
Rosa Virginia Hernández ${ }^{1}$ \\ Víctor Julio Useche Arciniegas ${ }^{2}$ \\ Luis Fernando Mariño 3 \\ UFPS
}

\section{RESUMEN}

El estudio tuvo como objetivo analizar los niveles de razonamiento manifestado por un grupo de estudiantes de secundaria cuando resuelven problemas que involucraran los conceptos de polígonos y poliedros siguiendo el modelo Van Hiele. Para el desarrollo de la investigación se optó por un enfoque cuantitativo, con un alcance descriptivo. Como técnicas para recolectar la información se diseñó e implementó un banco de preguntas, utilizando el software educativo

1 Magister en Educación Matemática. Licenciada en Matemáticas y Computación. Profesora adscrita al Departamento de Matemáticas y Estadística de la Universidad Francisco de Paula Santander (Cúcuta). Correo. rosavirginia@ ufps.edu. Orcid: /orcid.org/0000-0002-2638-671X

2 Maestría en Física. Ingeniero Civil. Profesor adscrito al Departamento de Física de la Universidad Francisco de Paula Santander (Cúcuta). Correo. victorjulioua@ufps.edu. co. Orcid: /orcid.org/0000-0003-0635-6676

3 Doctor en Educación Matemática. Magister en Educación Matemática. Licenciado en Matemáticas y Computación. Profesor Departamento de Matemáticas y Estadística de la Universidad Francisco de Paula. Correo. fernandomI@ufps.edu.co. Orcid: https://orcid.org/00000002-3438-6963
Viaje por la Geometría. Los resultados permitieron evidenciar un buen nivel de razonamiento alcanzado por los estudiantes al reconocer, analizar, clasificar, calcular el número de diagonales de un polígono y de utilizar el teorema de Euler para establecer la relación ente el número de caras, vértices y aristas del poliedro. En el proceso de razonamiento de deducción formal correspondiente al nivel cuatro no fue alcanzado por la gran mayoría de los estudiantes debido a la dificultad al realizar los cálculos algebraicos para calcular la apotema de un polígono en función de su lado; igualmente presentaron dificultad al calcular la diagonal del paralelepípedo.

PALABRAS CLAVE: Análisis Geométrico, Modelo de Van Hiele, Aprendizaje de polígono y poliedros 


\section{ABSTRACT}

The objective of this study was to analyze the levels of reasoning manifested by a group of high school students when solving problems involving the concepts of polygons and polyhedra following the Van Hiele model. For the development of the research, a quantitative approach was chosen, with a descriptive scope. A bank of questions was designed and implemented as techniques to collect the information, using the educational software Viaje por la Geometría (Journey through Geometry). The results showed a good level of reasoning achieved by the students in recognizing, analyzing, classifying, calculating the number of diagonals of a polygon and using Euler's theorem to establish the relationship between the number of faces, vertices and edges of the polyhedron. The formal deduction reasoning process corresponding to level four was not achieved by most of the students due to the difficulty in performing algebraic calculations to calculate the apothem of a polygon as a function of its side; they also had difficulty in calculating the diagonal of the parallelepiped.

Key words: Geometric analysis, Van Hiele's model, Polygon and polyhedra learning.

\section{INTRODUCCIÓN}

La geometría es una rama de las matemáticas que debe aprenderse en las escuelas, constituye una importante fuente de modelación y un ámbito por excelencia para desarrollar el pensamiento espacial y en particular, las formas diversas de argumentación sobre figuras bidimensionales y tridimensionales (Khasanah, Usado y Subanti, 2018). La comprensión de los modelos geométricos proporciona a los estudiantes habilidades de visualización y de razonamiento para resolver problemas del contexto (Rohendi, Septían y Sutarno, 2018).

Acorde con Al-ebous (2016) uno de los objetivos de la enseñanza de las matemáticas es mejorar los niveles de pensamiento geométrico en los estudiantes. Por esa razón, el profesor tiene un papel importante en el aprendizaje de la geometría, utilizando como estrategias herramientas tecnológicas que permitan el debate, exploración y participación en el salón de clase para que el estudiante pueda alcanzar los objetivos cognitivos y destrezas en los conceptos geométricos en cada nivel de estudio escolar.

Como lo afirmaAl-ebous (2016) una de las teorías que más ayuda a la enseñanza de la geometría es el modelo de razonamiento geométrico de Van Hiele, que ha traído la atención de investigadores y pedagogos de todo el mundo, puesto que ayuda a enseñar la geometría de forma eficaz en las etapas escolares a través de cinco fases: formación, orientación dirigida, explicación y orientación libre. Además, este modelo permite identificar la problemática enmarcada en la concepción que tienen los estudiantes hacia el aprendizaje de la geometría, repitiendo el proceso de los cinco niveles para identificar la evolución del razonamiento geométrico de cada individuo a través del: reconocimiento, análisis, clasificación, deducción formal, rigor de sólidos y volúmenes (Mulyayunita y Nurjanah, 2020).

Los esposos Pierre y Diana Van Hiele identificaron las dificultades del aprendizaje de la geometría evidenciadas por los estudiantes. Los investigadores desarrollaron una teoría que involucra niveles de razonamiento geométrico para que los estudiantes desarrollen su pensamiento geométrico a medida que progresan en su aprendizaje, partiendo del reconocimiento de una simple figura hasta poder describir una demostración geométrica formal (González, et ál., 2019).

Además, la enseñanza de la geometría se ve afectada por la metodología tradicionalista del profesorado, caracterizada por el énfasis en el discurso matemático como centro de enseñanza, 
en contravía de un aprendizaje significativo para el estudiante (Herbst, Cheah, Richard y Jones, 2018). En particular, los conceptos geométricos tridimensionales son poco enseñados y los libros de texto tradicionalmente utilizados están enfocados hacia el cálculo de perímetros, áreas de las figuras planas, dejando de lado las representaciones reales que permitan identificar conceptos fundamentales en la geometría como los poliedros y polígonos, su clasificación y los elementos que lo componen (Yudianto, Sugiarti y Trapsilasiwi, 2018).

El rendimiento de los estudiantes en el pensamiento espacial y sistema geométrico reflejado en los resultados de las pruebas saber (evaluaciones estandarizadas aplicadas por el Instituto Colombiano para la Evaluación de la Educación -ICFES) son muy bajos. Las razones son de diversa índole y esto refleja el desempeño del estudiante en el aula de clase, así como las estrategias didácticas usadas para abordar los contenidos establecidos para el aprendizaje de la Geometría (González, et ál., 2019). Desde hace más de una década, el Ministerio de Educación Nacional - MEN en Colombia estableció directrices para potenciar el pensamiento geométrico; sin embargo, se continúan impartiendo la enseñanza tradicionalista, y en el peor de los casos, algunos profesores no incluyen los contenidos de la geometría en las aulas de clase (Avila Rojas, 2019).

Actualmente, el MEN en Colombia, resalta la necesidad de investigar y buscar nuevos horizontes en la enseñanza de la geometría, donde se oriente al estudiante en la resolución de problemas geométricos del contexto (Bocanegra González y Devia Ávila, 2019). Una alternativa es el uso de herramientas como el software educativo libre o pago que posibilita la construcción de modelos geométricos y el razonamiento espacial en el estudiante, pero parece ser que los profesores lo siguen ignorando. Por tanto, se requiere que el profesor diseñe secuencias de actividades didácticas para crear ambientes, donde el estudiante construya bases sólidas y pueda ampliar sus conocimientos en geometría en los siguientes años escolares (Santacruz Rodríguez y Sacristán Rock, 2019).

Teniendo en cuenta la importancia de la geometría y la problemática en los procesos de su enseñanza y aprendizaje, la investigación se propuso responder la pregunta de investigación: ¿Cuál es el nivel de razonamiento según el modelo de Van Hiele, alcanzado por los estudiantes de grado noveno en el aprendizaje de los conceptos de poliedros y polígonos?

\section{MARCO CONCEPTUAL}

La investigación tuvo como fundamentos teóricos, los conceptos y definiciones tanto de polígonos como poliedros, así como los niveles de razonamiento que caracterizan el modelo de Van Hiele, además del pensamiento espacial y sistemas geométricos.

\subsection{Pensamiento espacial y los sistemas geométricos}

La palabra geometría se deriva de los vocablos griegos geos, que significa tierra y metrón que significa medida. Es tan antigua que fue estudiada por los egipcios, chinos, sumerios, romanos y griegos. Quienes la utilizaron en diferentes actividades, como la astronomía, la arquitectura, la agrimensura y la navegación. A pesar de ser una actividad recurrente y de sumo interés para las culturas antiguas, el nombre como tal llegaría un poco más tarde, exactamente en 1799, cuando el revolucionario francés de origen humilde, Gaspard Monge, expone con exactitud, en su obra, los diseños en dos dimensiones de objetos que tienen tres. Sin embargo, la geometría descriptiva existía desde hace mucho tiempo atrás, como lo muestra un grabado sumerio de una planta del templo de 
Ningirsú que data del año 2500 AC. atribuido a Gudea, rey arquitecto de Sirpurla en donde se manifiesta la capacidad de los dibujantes de la época para representar las características físicas de un edificio, que fuera interpretado por sus constructores (Colombo, Llanos y Otero, 2016).

La geometría puede verse como una ciencia del espacio y la forma para describir y medir figuras, construir teorías y estudiar modelos idealizados del mundo físico o de fenómenos que acontece el mundo real (Bonilla, Camargo, Castiblanco y Vanegas, 2012). Como lo expone Sotomontes, Bernal y González, (2019) el pensamiento espacial involucra procesos cognitivos que ayudan a construir y manipular las representaciones mentales de los objetos, las relaciones, transformaciones en un espacio determinado y su representación material.

\subsection{Definición y clasificación de polígonos y poliedros}

La Geometría estudia las formas de las figuras y los cuerpos geométricos. Los poliedros se pueden clasificar según su número de caras, según sus caras, según su regularidad y en convexos y cóncavos.

\subsubsection{Los polígonos}

El origen etimológico del término polígono es griego y se encuentra en la unión de dos vocablos: poli, que puede traducirse como "muchos", y "gono" que es sinónimo de muchos ángulos. Un polígono es la figura geométrica de un plano caracterizado por líneas rectas. Se trata de fragmentos consecutivos, sin alineación que reciben el nombre de lados. Existen diversas clasificaciones de los polígonos:

Polígono simple, es aquel en el que dos de sus aristas no consecutivas no se instersectan.

Polígono complejo, en cambio, tiene dos de sus aristas que no son consecutivas si se cortan.

Polígono cóncavo, es cuando lo atraviesa una recta y puede cortarlo en más de un punto.

Polígono convexo, es al ser atravesado por una línea recta, lo interrumpe en no más de dos puntos.

Polígono irregular, sus lados y ángulos son iguales

Polígono equiángulo, todos sus ángulos resultan iguales

Polígono equilátero, todos sus lados cumplen con la propiedad de la igualdad.

En cuanto a la forma de sus lados, los polígonos pueden se rectilíneos o curvilíneos. Cuando un polígono tiene más de dos dimensiones, por otra parte, puede denominarse poliedro (Godino y Ruíz, 2002, p. 462) 


\subsubsection{Los poliedros}

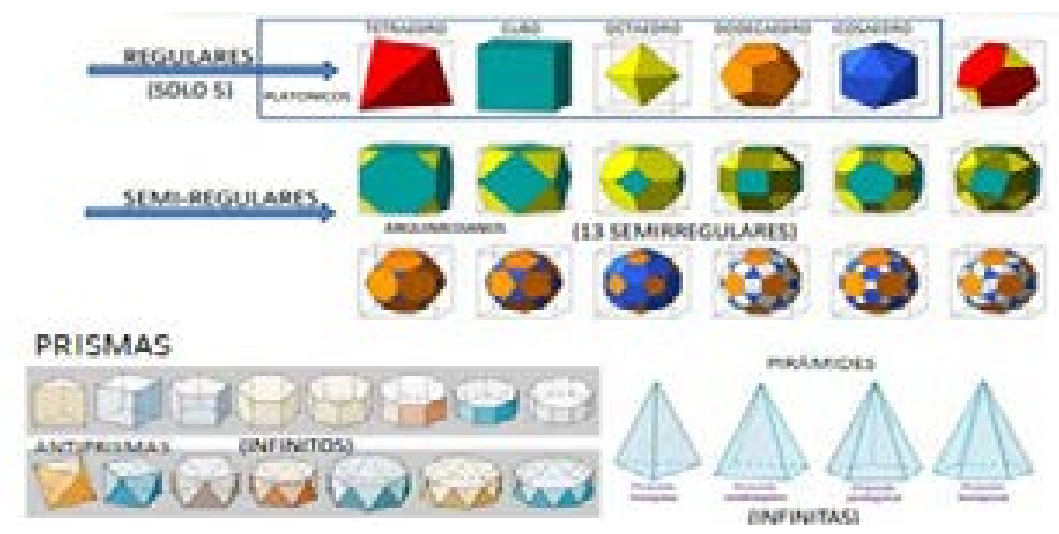

Figura 1. Poliedros con grados de regularidad. Fuente: (Sillero, 2018)

Los poliedros son entes geométricos que se destacan en distintos contextos como: en matemáticas, arquitectura, ingeniería, química y artes. Su presencia se impone en forma de templos, puentes, arcos, modelos de moléculas, gemas, esculturas, diseño gráfico, publicidad, decoración, paisajismo, coreografías, arreglos navideños y otras formas (Maríntez y Götte, 2010). Un poliedro es el sólido delimitado por una superficie cerrada simple formada por regiones planas. Cada región poligonal se dice que es un acara del poliedro, y lo vértices y lados de las regiones poligonales se dicen que son los vértices y lados del poliedro. Los poliedros fueron estudiados por filósofos y matemáticos célebres como Platón, Euclides, Arquímedes, Kepler, Poincaré, Hilbert, Coxeter (Godino y Ruíz, 2002, pág. 482).

Rojas (2016) define un poliedro como un sólido delimitado por una superficie cerrada simple formada por regiones poligonales planas. Cada región poligonal se dice que es una cara del poliedro, y los vértices y lados de las regiones poligonales se dicen que son los vértices y lados del poliedro. En la Naturaleza existen objetos con formas poliédricas. Por ejemplo, en cristalografía (cristales), biología (virus, radiolarios), las colmenas de las abejas en forma de rombododecaedros, la fachada hecha de celdillas hexagonales, etc.
Godino y Ruíz (2002) afirman que los poliedros fueron estudiados por filósofos y matemáticos célebres como Platón, Euclides, Arquímedes, Kepler, Poincaré, Hilbert y Coxeter. Un poliedro es la región encerrada o limitada por polígonos, cuyos elementos son:

Caras: cada uno de los polígonos.

Aristas: cada uno de los lados de los polígonos.

Vértices: cada uno de los puntos en los que se cortan las aristas.

\subsubsection{Características de los poliedros}

Cruz, Mántica y Götte (2015) utilizaron la cátedra Geometría Euclídea Espacial, afirmando que inicialmente se tiene cierta organización de los poliedros que nos permiten separarlos por determinadas características:

- Poliedro convexo: todos sus ángulos poliedros son convexos; si un poliedro es convexo, todas sus caras se podrán apoyar sobre un plano.

- Poliedro cóncavo: alguno de sus ángulos poliedros es cóncavo; si un poliedro es cóncavo, algunas de sus caras no podrán apoyarse en un plano. 
- Poliedros regulares: Se considera un poliedro regular cuando sus caras son polígonos regulares e iguales entre sí, y en cada vértice concurre el mismo número de aristas. Un poliedro regular es un poliedro con las siguientes características: la superficie es convexa, las caras son regiones poligonales regulares congruentes, concurren el mismo número de caras en cada uno de los vértices.

\subsection{El modelo de Van Hiele}

Según Mora y Rodríguez (2015), la teoría de Van Hiele fue creada en la escuela holandesa por los profesores Pierre Van Hiele y su esposa Dina Van Hiele-Geldof, en la década de los años cincuenta del siglo XX. La teoría está formada por dos componentes: el primero es la descripción de los distintos tipos de razonamiento geométrico de los estudiantes a lo largo de su formación matemática, que parte del razonamiento intuitivo de los niños hasta el formal y abstracto de los estudiantes; el segundo es una descripción de cómo puede un profesor organizar actividades de aula para que los estudiantes puedan alcanzar el nivel de razonamiento superior (Guillén, 2004).

Gutiérrez y Jaime (1991) consideraron que existen diferentes niveles o etapas de entendimiento de las ideas geométricas $y$, por esta razón, describen las características de cada fase o etapa del proceso de instrucción para permitirle al estudiante alcanzar un nivel de razonamiento superior al que tiene actualmente. Para Fouz y De Donosti, (2005), el modelo Van Hiele permite desarrollar e identificar los niveles de pensamiento y conocimiento de los estudiantes en las temáticas relacionados con el pensamiento espacial y sistemas geométricos. La Figura 1, sintetiza de forma sencilla los niveles de pensamiento geométrico planteados por Van Hiele, la teoría considera que cada uno de los niveles de pensamiento geométrico tiene sus propios símbolos lingüísticos y su propia red de relaciones que conectan esos símbolos.

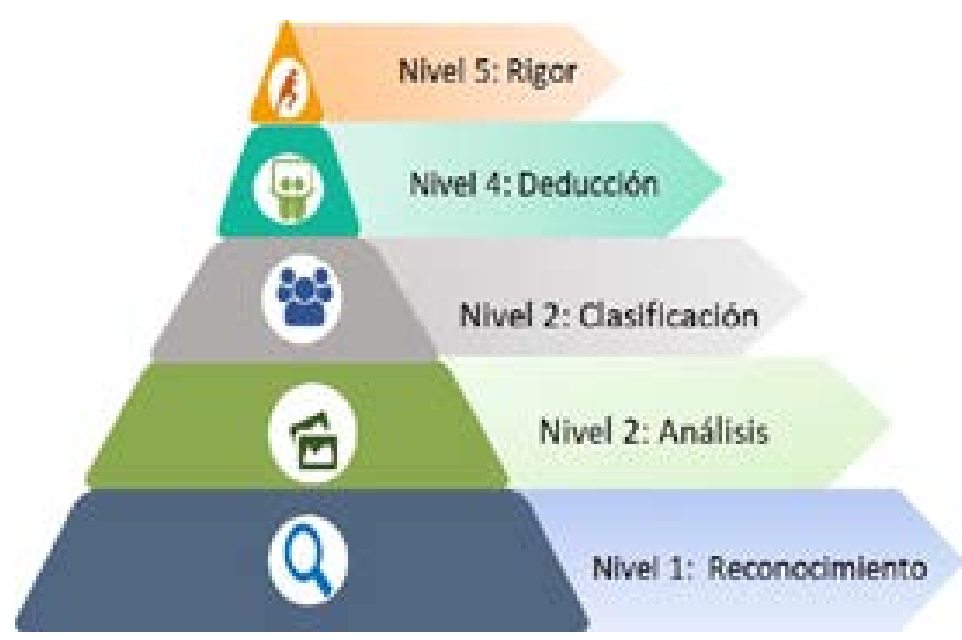

Figura 2. Niveles de Razonamiento de Van Hiele

La definición original del modelo de Van Hiele planteaba la existencia de cinco niveles de razonamiento; sin embargo, Guillen (2004) afirma que no se ha presentado unanimidad para aceptar el número de niveles. El mismo Van Hiele, como consecuencia del proceso de evaluación de sus ideas, en sus descripciones del modelo ha modificado en varias ocasiones la cantidad de niveles de razonamiento y sus características. Por lo tanto, para el desarrollo de la investigación y acorde con Vargas y Araya (2013), se analizará el razonamiento de los niveles 1, 2, 3 y 4 cuando el estudiante adquiere habilidades para resolver problemas de poliedros:

Nivel 1: Reconocimiento (Visualización). Los estudiantes perciben las figuras geométricas globalmente por su forma y no por sus propiedades. El proceso de razonamiento sobre conceptos básicos (formas o figuras simples) se lleva a cabo mediante consideraciones visuales de los objetos como un todo (Burger y Shaughnessy, 1986). Las figuras geométricas tales como cuadrados, triángulos, rectángulos, circunferencias, se perciben en su totalidad como una unidad, sin diferenciar sus propiedades 
y componentes (Vojkuvkova, 2012). En este nivel, el estudiante no es capaz de reconocer o explicar las propiedades determinantes de las figuras, no hay un lenguaje geométrico básico para referirse a figuras geométricas por su nombre (Vargas y Araya, 2013).

Nivel2: Análisis. Los alumnos pueden reconocer $y$ analizar las partes y propiedades de las figuras geométricas a través de la observación como de la experimentación (Fouz y De Donosti, 2005), pero no le es posible establecer relaciones o clasificaciones entre propiedades de distintas familias de figuras (Nunda, Graus y Henríquez, 2017).

Nivel 3: Clasificación (Abstracción). Para Alfonso (2004) los estudiantes comienzan a desarrollar su capacidad de razonamiento matemático. Establece las condiciones necesarias y suficientes que deben cumplir las figuras geométricas (Nunda, Graus y Henríquez, 2017), reconoce cómo unas propiedades se derivan de otras, estableciendo sus relaciones y clasificaciones lógicas. Siguen las demostraciones, pero en la mayoría de los casos no las entienden, esta carencia le impide captar la naturaleza axiomática de la geometría (Fouz y De Donosti, 2005).

Nivel 4: Deducción formal (Deducción). Para Alfonso (2004) los estudiantes pueden realizar razonamientos lógicos formales; las demostraciones de varios pasos ya tienen sentido para ellos, comprendiendo cómo se puede llegar a los mismos resultados partiendo de proposiciones o premisas distintas (Nunda, Graus y Henríquez, 2017). El estudiante está en capacidad de entender la naturaleza axiomática de las matemáticas realizando deducciones y demostraciones lógicas y formales para justificar las proposiciones planteadas y poder llegar a los resultados de proposiciones y premisas distintas (Fouz y De Donosti, 2005).
Nivel 5: Rigor. Según Alfonso (2004) los alumnos son capaces de trabajar en distintos sistemas axiomáticos, prescindiendo de cualquier soporte concreto para desarrollar su actividad matemática. Para Fouz y De Donosti (2005), el estudiante está en capacidad de desarrollar ejercicios de manera abstracta sin necesidad de ejemplos concretos. Este último nivel, por su grado de abstracción es el que menos investigaciones ha promovido (Alfonso, 2004).

\section{METODOLOGÍA}

Para dar respuesta a la pregunta de investigación ¿Cuál es el nivel de razonamiento según el modelo de Van Hiele, alcanzado por los estudiantes de grado noveno en el aprendizaje de los conceptos de poliedros y polígonos?, se optó por un enfoque cuantitativo, con un alcance descriptivo. Según Hernández-Sampieri y Torres, (2018) estos enfoques constitiuyen acercamientos iniciales a temas y fenómenos donde hay escaso conocimiento previo, o que han sido poco abordados.

\subsection{Población}

La población estuvo conformada por un grupo de estudiantes del grado noveno de educación básica del Instituto Técnico Alfonso López, de carácter público en el municipio de Ocaña (departamento de Norte de Santander), Colombia. La edad de los estudiantes está comprendida entre los 13 y 14 años. El 68\% de los estudiantes que participaron en la investigación son de género femenino.

\subsection{Diseño de la Investigación}

Acorde con Rodríguez-Rodríguez (2010) el componente didáctico del software educativo llamado Viaje por la Geometría se ha dado a conocer como un diseño instruccional de la metodología a usar y del tipo de evaluación a emplear, en relación con las características 
de los estudiantes y otras condiciones. Por lo tanto, para el desarrollo de la investigación, se implementó un software educativo diseñado con intervenciones de actividades didácticas a través de juegos interactivos.

Cada juego estaba conformado por un banco de 16 preguntas aleatorias, que permitieron identificar el nivel de razonamiento alcanzado por cada estudiante en el aprendizaje de los poliedros. Cabe notar que el software educativo no le permitía al estudiante pasar de un nivela a otro nivel de razonamiento, hasta tanto no haya logrado responder correctamente todas las preguntas correspondientes al nivel donde se encuentra.

El estudiante para iniciar el software debe aceptar el reto y comenzar a desarrollar la activad. Al terminar de interactuar el estudiante con el software educativo aparecerá un mensaje que dice ¡Felicidades por haber terminado el juego, puede repetirlo y así mejorar los resultados para alcanzar un mejor nivel! Los investigadores evalúan y analizan los resultados alcanzados por los estudiantes al resolver problemas de poliedros cuando utilizan el software educativo, identificando cuantos estudiantes pasan en cada nivel de razonamiento teniendo en cuenta el número de intentos; de esos intentos cuantas respuestas son correctas y cuantas incorrectas.

\subsection{Modelo de preguntas por nivel de razonamiento según el modelo de Van Hiele}

Siguiendo a Vargas y Araya (2013), la geometría constituye una importante fuente de modelación y un ámbito por excelencia para desarrollar el pensamiento espacial, y en particular, formas diversas de argumentación sobre figuras bidimensionales y tridimensionales. Para el proceso de análisis y descripción del nivel de razonamiento de los estudiantes de secundaria en la resolución de problemas de poliedros basado en el modelo de Van Hiele, se elaboró un banco de 16 preguntas aleatorias de los niveles 1, 2, 3 y 4 . A continuación, se presenta un modelo de cada pregunta por cada nivel:

Pregunta Nivel 1. Reconocimiento: ¿Qué forma tienen los recipientes?

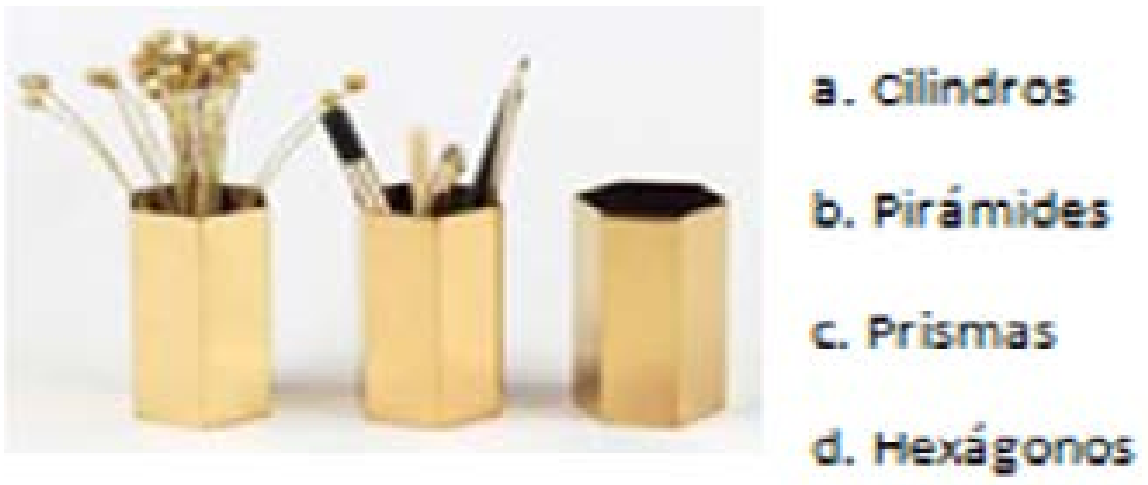

Figura 5. Pregunta de Nivel 1.

\section{Pregunta Nivel 2. Análisis:}

¿En un polígono la apotema es?

a. Un segmento que une el centro con uno de los vértices c. Un punto central que equidista de todos los vértices

d. Un segmento perpendicular a un lado desde el centro

b. Un segmento que forma el polígono 


\section{Pregunta Nivel 3. Clasificación:}

Una pirámide tiene 6 caras, 12 aristas y 8 vértices. ¿Aqué clase de pirámide corresponde?

a. Pirámide hexagonal

b. Pirámide octagonal truncado

c. Pirámide octagonal

d. Pirámide cuadrada truncado

\section{Pregunta Nivel 4. Deducción:}

Para calcular la diagonal de un ortoedro se debe:

Hallar el área del triángulo rectángulo

a. Hallar el valor de la diagonal d, en el triángulo

b. Determinar la diagonal de la base para utilizarla como cateto del triángulo rectángulo

c. Hallar el volumen del ortoedro y dividirlo entre dos

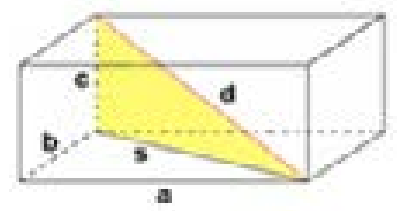

\section{RESULTADOS}

Se analizaron los resultados obtenidos en el aprendizaje de polígonos y poliedros por los estudiantes de grado noveno de educación básica, al desarrollar las actividades didácticas y la resolución de problemas basado en la teoría de Van Hiele. Acorde con Fouz y De Donosti (2005), se prescindirá para el presente estudio, el Nivel 5 denominado Rigor, debido a que la formación básica según los estándares curriculares establecidos por el Ministerio de Educación en Colombia para los grados octavos y noveno en la asignatura de geometría, contempla los procesos cognitivos mediante los cuales se construyen y se desarrolla un razonamiento de los objetos del espacio, las relaciones entre ellos, sus transformaciones y sus diversas representaciones. Por lo tanto, no se espera que el estudiante establezca y compare teoremas axiomáticos correspondientes al Nivel 5 .

La tabla 1 presentan los objetivos que se espera, logre cumplir el estudiante por niveles en el aprendizaje de Polígonos y Poliedros, siguiendo el modelo de Van Hiele. 
Tabla 1. Objetivos planteados por niveles de razonamiento, según el modelo de Van Hiele

\begin{tabular}{|c|c|c|c|c|}
\hline Contenido & Nivel 1 & Nivel 2 & Nivel 3 & Nivel 4 \\
\hline Polígonos & $\begin{array}{l}\text { Identificar figuras } \\
\text { que representa } \\
\text { polígonos }\end{array}$ & $\begin{array}{c}\text { Clasificar las figuras } \\
\text { en polígonos } \\
\text { regulares, convexos y } \\
\text { cóncavos. } \\
\text { Identificar los } \\
\text { elementos de un } \\
\text { polígono }\end{array}$ & $\begin{array}{c}\text { Calcular el } \\
\text { número de } \\
\text { diagonales de un } \\
\text { polígono }\end{array}$ & $\begin{array}{l}\text { Hallar la expresión } \\
\text { algebraica que } \\
\text { represente la apotema } \\
\text { de un polígono en } \\
\text { función del lado. }\end{array}$ \\
\hline Poliedros & $\begin{array}{l}\text { Reconocer } \\
\text { poliedros } \\
\text { y cuerpos } \\
\text { geométricos }\end{array}$ & $\begin{array}{l}\text { Clasificar las figuras } \\
\text { en poliedros: } \\
\text { regulares, irregulares, } \\
\text { convexos y cóncavos. } \\
\text { Identificar los } \\
\text { elementos de un } \\
\text { poliedro regular. }\end{array}$ & $\begin{array}{l}\text { Utilizar el } \\
\text { teorema de Euler } \\
\text { para establecer } \\
\text { la relación entre } \\
\text { el número de } \\
\text { caras, vértices } \\
\text { y aristas del } \\
\text { poliedro. } \\
\text { Identificar el } \\
\text { número de caras } \\
\text { que convergen } \\
\text { en un vértice. } \\
\text { Reconocer y } \\
\text { justificar que } \\
\text { el valor de las } \\
\text { sumas de los } \\
\text { ángulos de } \\
\text { las caras que } \\
\text { concurren en un } \\
\text { vértice es menor } \\
\text { que } 360^{\circ} \text {. }\end{array}$ & $\begin{array}{l}\text { Reconocer el } \\
\text { proceso que permite } \\
\text { hallar expresiones } \\
\text { algebraicas para } \\
\text { calcular la diagonal de } \\
\text { un paralelepípedo } \\
\text { Verifica expresiones } \\
\text { algebraicas para } \\
\text { calcular el área y } \\
\text { volumen del prisma } \\
\text { y pirámides y a partir } \\
\text { ellas deducir otros } \\
\text { poliedros. }\end{array}$ \\
\hline
\end{tabular}

La teoría de Van Hiele (1999) establece que el logro de una nueva etapa de comprensión no puede llevarse a cabo a través de la enseñanza de hechos y procedimientos, sino que el profesor debe crear un escenario favorable para que el estudiante alcance un nivel mayor de comprensión mediante una elección adecuada de actividades que representen un reto intelectual. Los niveles de razonamiento geométrico son progresivos y jerarquizados, esto significa que el estudiante al interactuar con el software educativo no tenía acceso al siguiente nivel hasta que no respondiera correctamente las preguntas del nivel en donde se encontraba. Completar un determinado nivel significa que el estudiante desarrolló los procesos de razonamiento que caracterizan cada nivel. Por lo tanto, el aprendizaje de los polígonos y poliedros el estudiante debe transitar por cada uno de los niveles en el orden establecido. 
Tabla 2. Resultados de las actividades didácticas en el software educativo

\begin{tabular}{|c|c|c|c|r|}
\hline Nivel & Intentos & Correctas & Incorrectas & Total \\
\hline 1 & 227 & 171 & 56 & $85,70 \%$ \\
\hline 2 & 227 & 175 & 52 & $84,33 \%$ \\
\hline 3 & 397 & 222 & 175 & $66,78 \%$ \\
\hline 4 & 207 & 144 & 63 & $50,09 \%$ \\
\hline
\end{tabular}

La Tabla 2, presenta los resultados por cada nivel. Un $85,70 \%$ de los estudiantes lograron superar el nivel 1 con 227 intentos, alcanzando 171 respuestas correctas y 56 respuestas incorrectas. Mientras que en el cuarto nivel solo se alcanzó un $50 \%$ de los estudiantes con 207 intentos, obteniendo 144 respuestas correctas y 63 incorrectas.

Los estudiantes obtuvieron buenos resultados al identificar si la figura de un polígono y reconocieron los poliedrosycuerpos geométricos. Se observa que el menor porcentaje es el nivel 4; esto resultados permitieron identificar un bajo número de estudiantes que lograron adquirir la capacidad de deducir una expresión algebraica para calcular la apotema de un polígono en función del lado y también no se evidenció el proceso conceptual para calcular la diagonal de un paralelepípedo a través de expresiones algebraicas.

Tabla 3. Calificación total por niveles de las preguntas

\begin{tabular}{|ll|c|}
\hline Niveles de Razonamiento & Calificación \\
\hline 1. & Reconocimiento & 4,8 \\
2. & Análisis & 4,3 \\
3. & Clasificación & 4,1 \\
4. & Deducción & 1,6 \\
\hline
\end{tabular}

Para resolver el banco de preguntas en el software los estudiantes tenían que ir contestando e interactuando en cada actividad didáctica. Se logró un resultado muy satisfactorio con una nota de calificación de 4,8 (escala de 1,0 hasta 5,0 ) en el nivel 1 sobre el reconocimiento de las figuras de los polígonos y poliedros. En el nivel
2, algunos estudiantes presentaron dificultades en el proceso de clasificación de los polígonos y poliedros. El nivel 3 se obtuvo la calificación de 4.1 debido a que se presentaron dificultades en los estudiantes al calcular el número de diagonales en un polígono y utilizaron de forma incorrecta el teorema de Euler para establecer la relación entre el número de caras, vértices y aristas de un poliedro.

Finalmente se evidenció que el proceso de razonamiento de deducción del nivel cuarto fue el más bajo (con nota de 1,6) presentándose dificultades por la mayoría de los estudiantes para entender el proceso conceptual de un polígono en función de un lado al no poder calcular la expresión algebraica; igualmente, no calcularon la diagonal de un paralelepípedo al no reconocer el proceso para poder expresar el concepto algebraicamente.

\section{CONCLUSIONES}

Las respuestas manifestadas por los participantes, permitieron dar respuesta a la pregunta de investigación y al propósito del estudio acerca de describir los niveles de razonamiento de los estudiantes de secundaria cuando resolvieron problemas que involucraron los conceptos de polígonos y poliedros de acuerdo al modelo de Van Hiele. Los datos muestran que la gran mayoría de los estudiantes que participaron en el estudio desarrollaron los niveles de razonamiento 1 y 2 , al lograr en el software educativo Viaje por la Geometría, identificar cuál de las figuras es un polígono y un poliedro. Igualmente, se evidenció la 
habilidad y capacidad para clasificar las figuras en regulares, irregulares, convexos y cóncavos.

En el razonamiento del nivel 3 (Clasificación), el $20 \%$ de los estudiantes no alcanzaron a superarlo, se evidencian dificultades al utilizar el teorema de Euler que permite establecer la relación entre el número de caras, vértices y aristas del poliedro. En la deducción formal correspondiente al nivel 4 , solo fue alcanzada por el $30 \%$ de los estudiantes, de nuevo se evidencian dificultades de razonamiento para reconocer los procesos del desarrollo de la actividad en el software educativo, para poder hallar la expresión algebraica represente la apotema de un polígono en función de su lado y calcular la diagonal del paralelepípedo.

Queda como propuesta de estudio profundizar en los procesos de desarrollo en la resolución de problemas de los conceptos de polígonos y poliedros utilizando herramientas tecnológicas para que los estudiantes logren alcanzar la capacidad de entender la naturaleza axiomática de las matemáticas realizando deducciones lógicas y formales para justificar proposiciones planteadas en el contexto del aprendizaje de la geometría.

\section{REFERENCIAS BIBLIOGRÁFICAS}

Al-ebous, T. (2016). Effect of the Van Hiele Model in Geometric Concepts Acquisition: The Attitudes towards Geometry and Learning Transfer Effect of the First Three Grades Students in Jordan. International Education Studies, 9(4), 87-98.

Alfonso, M. (2004). Sobre los niveles de pensamiento geométrico de Van Hiele y la formación de profesores en activo. Revista de Didáctica de las Matemáticas, 58, 3-35.
Avila Rojas, O. (2019). Aprendizaje significativo en geometría para el grado octavo (Tesis de Maestría). Obtenido de Universidad Pedagógica y Tecnológica de Colombia: https://repositorio.uptc. edu.co/handle/001/2729

Bocanegra González, I. X., \& Devia Ávila, M. A. (2019). Construcción de una trayectoria hipotética de aprendizaje en torno al proceso de generalización geométrica. Obtenido de Universidad Pedagógica Nacional: http://hdl.handle. net/20.500.12209/11913

Bonilla, S., Camargo, L., Castiblanco, A. C., \& Vanegas, Y. M. (2012). Capitulo 4. Pensamiento Espacial y Sistemas Geométricos. En A. Asociación Colombiana de Matemática Educativa, Cuaderno $N^{\circ} 5$. Estándaes Curriculares. Área matemáticas. Aportes para el análisis (págs. 34-43). gaia.

Burger, W., \& Shaughnessy, J. (1986). Characterizing the van Hiele levels of development in geometry. Journal for research in mathematics education, 17(1), 31-48.

Cruz, M. F., Mántica, A. M., \& Götte, M. (2015). Clasificaciones de poliedros en estudiantes del profesorado de matemática. IV Jornadas de Enseñanza e Investigación Educativa en el campo de las Ciencias Exactas y Naturales., 1-12.

Fouz, F., \& De Donosti, B. (2005). Modelo de Van Hiele para la didáctica de la Geometría. Un paseo por la geometría, 67-82.

Godino, J., \& Ruíz, F. (2002). Geometría y su didáctica para maestros. Universidad de Granada, Departamento de Didáctica. 
González, N., Chavarro, M., Mojica, C., \& Peña, C. (2019). La geometría, eje integrador del pensamiento matemático en educación básica. Educación y Ciencia, 23, 495-511.

Gutiérrez, Á., \& Jaime, A. (1991). El Modelo de razonamiento de Van Hiele como marco para el aprendizaje comprensivo de la geometría. Un ejemplo: Los Giros. Educación Matemática, 30(02), 49-65.

Herbst, P., Cheah, U., Richard, P., \& Jones, K. (2018). International Perspectives on the teaching and learning of geometry in secondary schools (Vol. 21). Springer International Publishing. Obtenido de doi.org/10.1080/14794802.2018.15310 55

Hernández-Sampieri, R., \& Torres, C. (2018). Metodología de la investigación (Vol. 4). México DF: McGraw-Hill Interamericana.

Khasanah, V., Usodo, B., \& Subanti, S. (2018). Guided discovery learning in geometry learning. Journal of Physics: Conference Series, 983(1), 1-6.

Maríntez, N., \& Götte, M. (2010). Exploración, Construcción y Clasificación de Poliedros. Obtenido de Facultad de Ciencias Exactas Ingeniería y Agronomía, Universidad Nacional de Rosario: https://www.fceia.unr. edu.ar/matematica/jornadas2010/ materiales/exploracion_construccion_ clasificacion_poliedros.pdf

Nunda, F., Graus, M., \& Henríquez, L. (2017). Estrategia Didáctica para el desarrollo del pensamiento geométrico en el proceso de enseñanza-aprendizaje de la matemática en la enseñanza primaria angoleña. Revista Científica Multidisciplinaria, 1(1), 75-88.
Rodríguez-Rodríguez, L. (21 de Junio de 2010). Concepción didáctica del software educativo como instrumento mediador para un aprendizaje desarrollador. Obtenido de Doctoral dissertation, Universidad de Ciencias Pedagógicas "Félix Varela Morales". Dirección de Tecnología Educativa: https://dspace. uclv.edu.cu/handle/123456789/8194

Rohendi, D., Septían, S., \& Sutarno, H. (2018). The use of geometry learning media based on augmented reality for junior high school students. IOP conference series: Materials science and engineering, 306(1), 1-7.

Rojas, C. (2016). Introducción a la geometría. Universidad del Norte.

Santacruz Rodríguez, M., \& Sacristán Rock, A. (2019). Una mirada al trabajo documental de un profesor de primaria al seleccionar recursos para enseñar geometría. Educación Matemática, 31(3), 7-38.

Sillero, D. (2018). Mundo Poliedros. Obtenido de Académica : https://www.academia. edu/42605593/MUNDO_POLIEDROS

Sotomontes, P., Bernal, A., \& González, A. (2019). Estrategias medidas por TIC para desarrollar el pensamiento espacial y los sistemas geométricos. Revista internacional de Aprendizaje en Ciencia, Matemática y Tecnología, 6(1), 21-27. Obtenido de https://doi.org/10.37467/ gka-revedumat.v6.1879

Van Hiele, P. (1999). Developing geometric thinking through activities that begin with play. Teaching children mathematics, 5(6), 310-316. 
Vargas, G., \& Araya, R. (2013). El modelo de Van Hiele y la enseñanza de la geometría. Uniciencia, 27(1), 74-94.

Vojkuvkova, I. (2012). The van Hiele model of geometric thinking. WDS'12 Proceedings of Contributed Papers, 1, 72-75.

Yudianto, E., Sugiarti, T., \& Trapsilasiwi, D. (2018). La identificación de estudiantes de nivel van Hiele sobre el tema de la geometría analítica espacial. En Journal of Physics: Conference Series, 983(1), 1-7. 